\title{
The coherence of powerful laser diodes and formation of lasing channels
}

\author{
Alexey Rzhanov* \\ Lomonosov Moscow State University, Faculty of Physics, 119991 Moscow, Russia
}

\begin{abstract}
The effect of the radiation coherence length on the lasing channels lateral dimensions in a planar laser diode with a wide contact is considered. The relationship between the coherence of high-power laser diode radiation, its spectrum and the nonlinear optical properties of the laser resonator active medium is discussed.
\end{abstract}

\section{Introduction}

Laser diodes (LD) with a wide contact (more than 50 microns) have a characteristic peculiarity: in their lateral mode spectrum, as a rule, there are non-phase-locked spatial channels of generation $[1,2]$. The appearance of strong nonlinearities that lead to the filamentation of laser radiation at low radiation power is primarily caused by the imperfection of the layer growth technology. Modern LD with a wide contact and quantum wells $(\mathrm{QW})$ show the similar modes of generation, but it occurs at high power levels $(0.5 \mathrm{~W}$ and more) [3]. Physical mechanisms causing the filamentation in LD with a wide contact are the same that were previously. But LD with QW has a radiation spectrum of 2-3 orders narrower, and the gain much higher at the same pump. This circumstance leads to the necessity to take into account the influence of nonlinear refraction and light spatial coherence on the spectral-spatial characteristics of $\operatorname{LD}$ radiation $[4,5]$.

\section{Laser diodes modeling}

The first work on modeling processes in LD appeared in the 80-ies years of the last century $[6,7]$. The modern basic model for the self-consistent interaction of radiation, pumping and multilayer semi-conductor structure is described, for example, in [8, 9]. All self-consistent models are based on kinetic and wave equations. In the distributed models of LD, the space-inhomogeneous interaction between the laser radiation and non-equilibrium carriers is taken into account. The nonlinearity of refraction and amplification of LD active medium in distributed models is considered in the form of the effective permittivity dependence on the non-equilibrium carriers concentration $[9,10]$. However, this approach is not applicable for LD with a wide contact, because of the only wave equation is taken for determining the laser field modes [9]. An approximation is considered for a single set of modes in the lateral

\footnotetext{
* Corresponding author: rjanov@mail.ru
} 
limits of the entire waveguide. But in the case of LD with a wide contact we must consider each radiation channel separately as has its own set of coherent modes.

The decay of an optical field on the channels in LD was demonstrated in [11]. In such devices, the threshold is exceeded both within a contact width (100-200 mkm) and in QW gain bandwidth (about $10 \mathrm{meV}$, or 4-6 nm). If LD radiation coherence length (4-5 $\mathrm{cm} \mathrm{[12,}$ 13]) is less than optical path, the lasing field splits into non-phased-locked channels. A spatial self-focusing caused by nonlinear refraction completes the picture.

\section{Results and discussion}

The above threshold regime implies in LD a dense filling of the modes in spectral gainband. This explains the separation of channels in the experimental spectrum $[11,14]$.

Taking into account the finite coherence length when using the diffraction theory gives the following estimate of the channel width for the fundamental transverse mode $w_{0}$ :

$$
w_{0}=\sqrt{\frac{\lambda L_{c o h}}{2 \pi n_{\text {eff } 0}}},
$$

where $\lambda$ and $L_{c o h}$ are the central wavelength and the coherence length of LD radiation in vacuum, $n_{\text {effo }}$ is the effective refractive index of the fundamental mode. This approximation is quiet consistent with the results of [7].

Consideration of a nonlinear medium with a carrier-induced quadratic refraction index profile under the assumption of almost ideal coherent radiation leads to the following estimation [15]:

$$
w_{0}=\frac{\lambda}{2 \pi n_{\text {eff } 0}} \sqrt{\frac{\lambda}{\Delta \lambda}},
$$

where $\Delta \lambda$ is a spectral fundamental mode linewidth.

Comparing the expressions (1) and (2), in the presence of experimental data, it is possible to estimate the magnitude of the relationship between the radiation coherence length and the nonlinear properties of the active medium in LD with a wide contact. So it is shown that the modeling of LD with a wide contact requires taking into account both the coherence and nonlinear properties that makes up induced waveguides.

\section{References}

1. Zh.I. Alferov, FTP, 32, 3 (1998)

2. A.S. Logginov, I.I. Vinogradov, Proc. of SPIE, 2513, 799 (1995)

3. I.S. Tarasov, Quant. Electron., 40, 8 (2010)

4. V.V. Bliznyuk, O.I. Koval, A.G. Rzhanov et al., Izv. RAN Ser. Fiz., 79, 1666 (2015)

5. A.E. Zhukov, Fundamentals of physics and technology of semiconductor lasers ( $\mathrm{SPb}$.: Ed. Academ. Univ., 2016)

6. J. Buus, IEEE J. Quant. Electron. QE-18, 7 (1982)

7. J. Buus, IEEE J. Quant. Electron. QE-19, 6 (1983)

8. A.G. Rzhanov, V.I. Gverdtsiteli, A.M. Arbash, RUDN J. Math. Inf. Phys. 3, 72 (2009)

9. A.G. Rzhanov, Izv. RAN Ser. Fiz., 82, 1 (2015)

10. H. Kogelnik, Guided-Wave Optoelectronics, ed. T. Tamir (Springer-Verlag, 1988)

11. O.I. Koval, A.G. Rzhanov, G.A. Solovyev, Phys. of WP, 21, 4 (2013)

12. S.P. S.P. Vorobjev, http://www.holography.ru/files/holmich.htm (2018)

13. A.V. Lukin, Opt. J. 79, 3 (2012)

14. V.V. Bliznyuk, O.I. Koval, A.G. Rzhanov at al., EPJ Web of Conf. 161, 03004 (2017)

15. A.G. Rzhanov, Izv. RAN Ser. Fiz., 83, 12 (2019) - (to be pubished). 